\title{
The prevalence and genotype distribution of rotavirus $A$ infection among children with acute gastroenteritis in Kunming, China
}

\author{
Ziqin Dian ${ }^{1,2} \cdot$ Mao Fan $^{3} \cdot$ Binghui Wang ${ }^{1} \cdot$ Yue Feng $^{1} \cdot{\text { Hao } \mathrm{Ji}^{4} \cdot \text { Shuwei Dong }}^{1} \cdot$ \\ A-Mei Zhang ${ }^{1} \cdot \mathrm{Li} \mathrm{Liu}^{1} \cdot \mathrm{Hua} \mathrm{Niu}^{2} \cdot \mathrm{Xueshan} \mathrm{Xia}^{1}{ }_{\mathbb{C}}$
}

Received: 13 August 2016/Accepted: 30 September 2016/Published online: 7 October 2016

(c) The Author(s) 2016. This article is published with open access at Springerlink.com

\begin{abstract}
The aim of this study was to determine the prevalence of rotavirus A (RVA) infections in children from Kunming, China, and the RVA genotypes present. A total of 16,311 children with acute gastroenteritis were recruited for the study, and $33.1 \%(5,394 / 16,311)$ were RVA positive. Children under 24 months of age were more susceptible to RVA infection, with an infection rate of $87.4 \%(4,712 / 5,394)$. The most prevalent genotype was G9P[8] (85/107, 79.4\%), which showed high sequence similarity to G9P[8] strains from other regions of China and neighbouring countries, but not to the licensed vaccine strain LLR. These findings should be useful for the prevention of RVA infections.
\end{abstract}

Keywords Rotavirus A · Children · Gastroenteritis · Epidemiology $\cdot$ Genotype

Z. Dian and M. Fan contribute to the article equally.

Electronic supplementary material The online version of this article (doi:10.1007/s00705-016-3102-6) contains supplementary material, which is available to authorized users.

Xueshan Xia

oliverxia2000@aliyun.com

1 Faculty of Environmental Science and Engineering, Faculty of Life Science and Technology, Kunming University of Science and Technology, 727 Jingming South Road, Kunming 650500, Yunnan, China

2 Department of Clinical Laboratory, First People's Hospital of Yunnan Province, Kunming 650032, Yunnan, China

3 Department of Clinical Laboratory, Kunming Children's Hospital, Kunming 650034, Yunnan, China

4 Department of Information Center, First People's Hospital of Yunnan province, Kunming 650032, Yunnan, China
Rotaviruses are important pathogens that cause acute gastroenteritis in both humans and animals. Rotavirus infections may lead to severe dehydration, electrolyte disorders, and acid-base disturbances. According to WHO estimates, the number of deaths of infected children worldwide in 2013 was 215,000 [1]. Rotaviruses belong to the family Reoviridae, whose members are characterized by a wheelshaped capsid structure [2]. The virion consists of a triplelayered protein capsid containing 11 segments of dsRNA encoding six structural proteins (VP1-VP4, VP6, and VP7) and five or six nonstructural proteins (NSP1-NSP6) [3]. Rotaviruses have been classified into different genotypes based on the sequences of VP7 (G-type antigen) and VP4 (P-type antigen, which is cleaved into VP5* and VP8* by trypsin). In the last 30 years, at least $27 \mathrm{G}$ and $37 \mathrm{P}$ genotypes have been reported [4], and combinations of G1, G2, G3, G4, G9, and G12 with P[4], P[6], and P[8] have been found in humans [5]. Based on the serological characterization of VP6, rotaviruses have been classified into eight different groups (A-H). Among these, group A (RVA) is the most important pathogen in human children [6].

In China, the RVA infection rates in children range from $28 \%$ to $65 \%$, and the hospitalization rate is $30-50 \%$ [7, 8]. The city of Kunming is located in the southwestern region of China, with an annual average temperature $15^{\circ} \mathrm{C}$ and a permanent resident population of 5,505,031. The population is composed of numerous ethnicities, and the economic and health status of the region is relatively poor. To date, few studies have reported molecular epidemiological data for RVA infections in Kunming [9]. In order to monitor the epidemiology of RVA and to estimate the effectiveness of the vaccine that is permitted for use in this area, the prevalence of RVA infection and genotypes among children with acute gastroenteritis were determined. 
From February 2015 to January 2016, a total of 16,311 children under the age of 14 years with acute gastroenteritis were recruited from two representative hospitals (First People's Hospital of Yunnan Province and Kunming Children's Hospital). Demographic information was recorded during sampling. RVA antigens were detected using a Rapid Chromatographic RotaA Diagnostic Kit (Wantai, Beijing, CHN). RVA-positive faecal specimens were diluted 1:10 with PBS (Vircell, ESP, pH7.2) and centrifuged (8,000 rpm, $30 \mathrm{~min})$. Then, the supernatants were stored at $-80{ }^{\circ} \mathrm{C}$. Subsequently, the viral genome was extracted from the supernatants using a TIANamp Virus RNA Kit (Tiangen Biotech, Beijing, CHN). RVA infection was confirmed by RT-PCR with VP6 primer [10] (Table S1).

In the present study, 120 samples were randomly selected from 5,394 RVA-positive specimens. Genotyping was performed using RT-nested PCR (RT-nPCR) with VP4 and VP7 primers (Table S1) [10, 11]. PCR products were purified using an Agarose Gel DNA Extraction Kit (Takara, Dalian, $\mathrm{CHN}$ ) for commercial sequencing (Tsingke, Kunming, $\mathrm{CHN}$ ). Sequences of VP7 and VP4 were submitted to GenBank under the accession numbers KX033584-KX033690 and KX033691-KX033797, respectively.

All sequences of VP7 and VP4 used in this study were aligned using the integrated Clustal X 1.83 programme, and neighbour-joining (NJ) trees were constructed in MEGA 6.0, using the Kimura 2-parameter model with gamma distribution and invariant sites as described previously [12]. The nodal reliability of the $\mathrm{NJ}$ trees was assessed by bootstrap (BS) analysis with 1000 pseudo-replicates; values below $70 \%$ were excluded as non-significant. The percentage of nucleotide sequence identity was calculated using an online tool (http://www.genome.jp/tools/clustalw/).

Of the faecal samples collected from the 16,311 recruited children with acute gastroenteritis, 5,394 samples were found to be RVA positive, indicating an infection rate of $33.1 \%$. RVA infections are known to occur at a lower rate in spring and summer, with an increased incidence in late autumn. In the present study, a higher occurrence of RVA infections was detected from November 2015 to January 2016, with a peak infection rate of up to $71.5 \%$ in November. However, the infection rate decreased to $6 \%$ $8 \%$ from July to September 2015 (Table S2), suggesting that the RVA infection rate was low and that gastroenteritis during this period was mainly caused by enterobacteria and enteric viruses, such as astrovirus, norovirus, and adenovirus [13]. Notably, children less than 2 years of age accounted for $87.4 \%(4,712 / 5,394)$ of the total infections in the study period (Table S3). The infection rate showed an overall declining trend with increasing age after 36 months. Although RVA infections were also found in 5- to 14-year-old children, these accounted for only $2.0 \%$ (110/ 5,394 ) of all positive samples (Table S3).
Among the 120 randomly selected RVA-positive specimens, VP7-encoding open reading frames (ORFs) and VP4 partial ORFs were successfully obtained from 107 $(107 / 120,89.2 \%)$ and $112(112 / 120,93.3 \%)$ samples, respectively. Mismatches between PCR primers and templates represent a possible reason for the failure of amplification of VP7 ORFs and partial VP4 ORFs. Five of 112 samples could not be genotyped with the VP7 primers, and this may be attributed to point mutations in the target regions [14], as has been reported previously [12].

The phylogenetic relationships of VP7 ORFs and VP4 partial ORFs to sequences from China and other countries were examined. Sequences of VP7 were separated into nine branches, and the present strains were classified under branches G1, G2, G3, G4, and G9. Eighty-nine G9 strains from this study were classified into two clusters with reference strains from China and neighbouring countries. Some strains formed several sub-clusters in one branch; however, km15060 was not related to other strains and formed an individual branch, which may imply the existence of a local endemic strain. Fifteen G2 strains formed two clusters with strains from China, Thailand, and Australia. The G3 and G4 branches consisted of strains from other regions of China and other countries; however, the G1 strain only clustered with foreign strains, which implied that this strain may have been imported into China (Fig. 1). Six genotypes, namely P[4], P[6], P[8], P[9], P[10], and $\mathrm{P}[14]$, were determined for VP4. Eighty-five P[8] strains with high nucleotide sequence similarity (98.6-100\% identity) formed cluster 1 with reference strains. Two strains, namely km15002 and km15009, were in cluster 2; the first clustered with strains from the USA, China and Japan, while the second clustered together with strains from Japan, Australia, Thailand, and Brazil. The latter group previously represented the most prevalent strains worldwide. Nineteen strains corresponding to the P[4] genotype were in the same cluster with foreign strains, and one P[6] strain was clustered with strains from China and other countries (Fig. 2).

Fifteen G2P[4] (15/107, 14.0 \%) strains were distributed in five groups of children, mainly in the 13- to 24-monthold group $(6 / 15,40 \%)$, the 25 - to 36-month-old group (4/ $15,26.7 \%)$, and the 37- to 48-month-old group (3/15, $20 \%$ ), but not in the youngest group. This strain was considered to mainly infect older children [15] and accounted for $46.7 \%(7 / 15)$ of infections in children 2 years of age. Four G9P[4] (4/107, $3.7 \%)$ strains were detected in one child in the 0 - to 12-month-old group, two in the 13- to 24-month-old group, and one in the 61- to 168-month-old group. The other genotypes included G1P[8] (1/107, $0.9 \%)$, G3P[8] (1/107, $0.9 \%)$, and G4P[6] (1/107, $0.9 \%)$, which were detected in children 36,13 and 20 months of age, respectively (Table 1). Although G1P[8] 


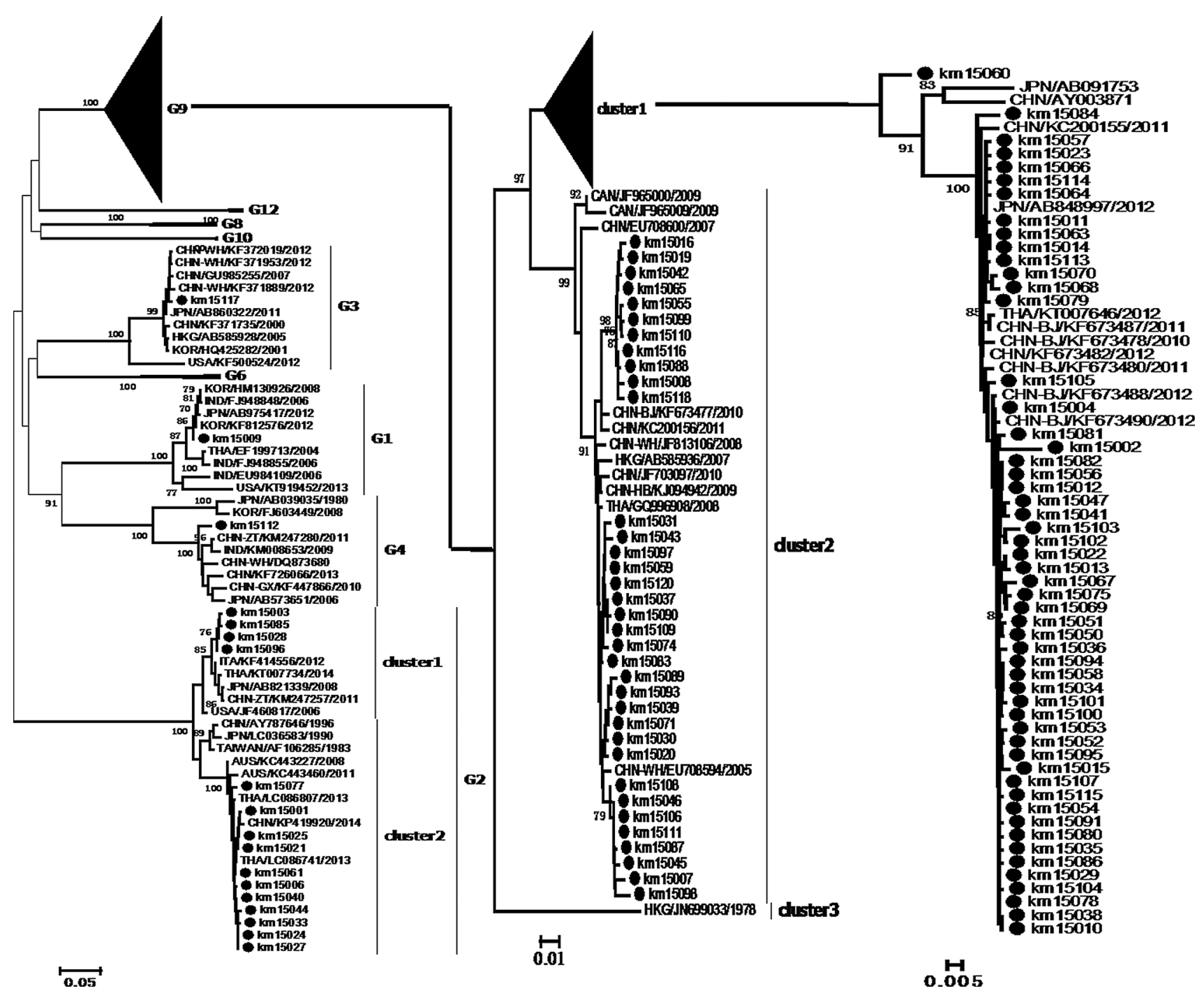

Fig. 1 Phylogenetic trees of VP7 sequences. A neighbor-joining (NJ) tree was constructed using MEGA 6.0 software with the Kimura 2-parameter model with gamma distribution. Bootstrap values were

and G3P[8] strains were once widespread in China $[5,16]$, these strains were detected at a lower frequency in Kunming in the present study. These findings suggest that the G1-G4 strains, which represented the most prevalent genotypes worldwide in the past, are no longer the dominant strains in this region $[17,18]$.

The distribution of the G9 strain is increasing worldwide. This genotype was first discovered in the USA in 1983 [19]; however, its prevalence was low for about a decade following its discovery. The distribution of the G9 strain increased worldwide after 2000 [20]. In China, the G9 strain was first identified in Yunnan province in 1998 [21]; only sporadic detections were reported in the following decade. By 2011, this genotype replaced the G1 and G3 strains and became the most prevalent genotype in Wuhan and Nanjing $[16,22]$. In this study, G9P[8] was the most predominant determined for 1000 repetitions. Kunming strains are indicated by black dots. The sequences of reference strains were obtained from GenBank

genotype, accounting for $79.4 \%$ (85/107) of all strains. The $\mathrm{G} 9 \mathrm{P}[8]$ genotype was also prevalent in all age groups, especially in 0 - to 12 -month-olds $(36 / 85,42.4 \%)$ and 13 - to 24-month-olds (38/85, $44.7 \%)$ (Table 1).

It has been suggested that vaccination programs should be mainly targeted at younger children [13]. The amino acid sequence similarity of the dominant G9P[8] strains in this study and three vaccine strains were analysed. Lanzhou lamb rotavirus vaccine (LLR), which is a G10P[15]-based monovalent live attenuated oral vaccine licensed in China, was used. The other two vaccines were Rotarix ${ }^{\mathrm{TM}}$ (G1P[8]) and RotaTeq ${ }^{\mathrm{TM}}$ (G1-G4P[8]), which are commonly used worldwide, but not in China. Amino acid residues on the surface of VP7 and VP4 that are responsible for neutralization were designated as 7-1a, 7-1b and 7-2, 8-1, 8-2, 8-3 and 8-4, respectively. By Clustal-W alignment of the VP7 


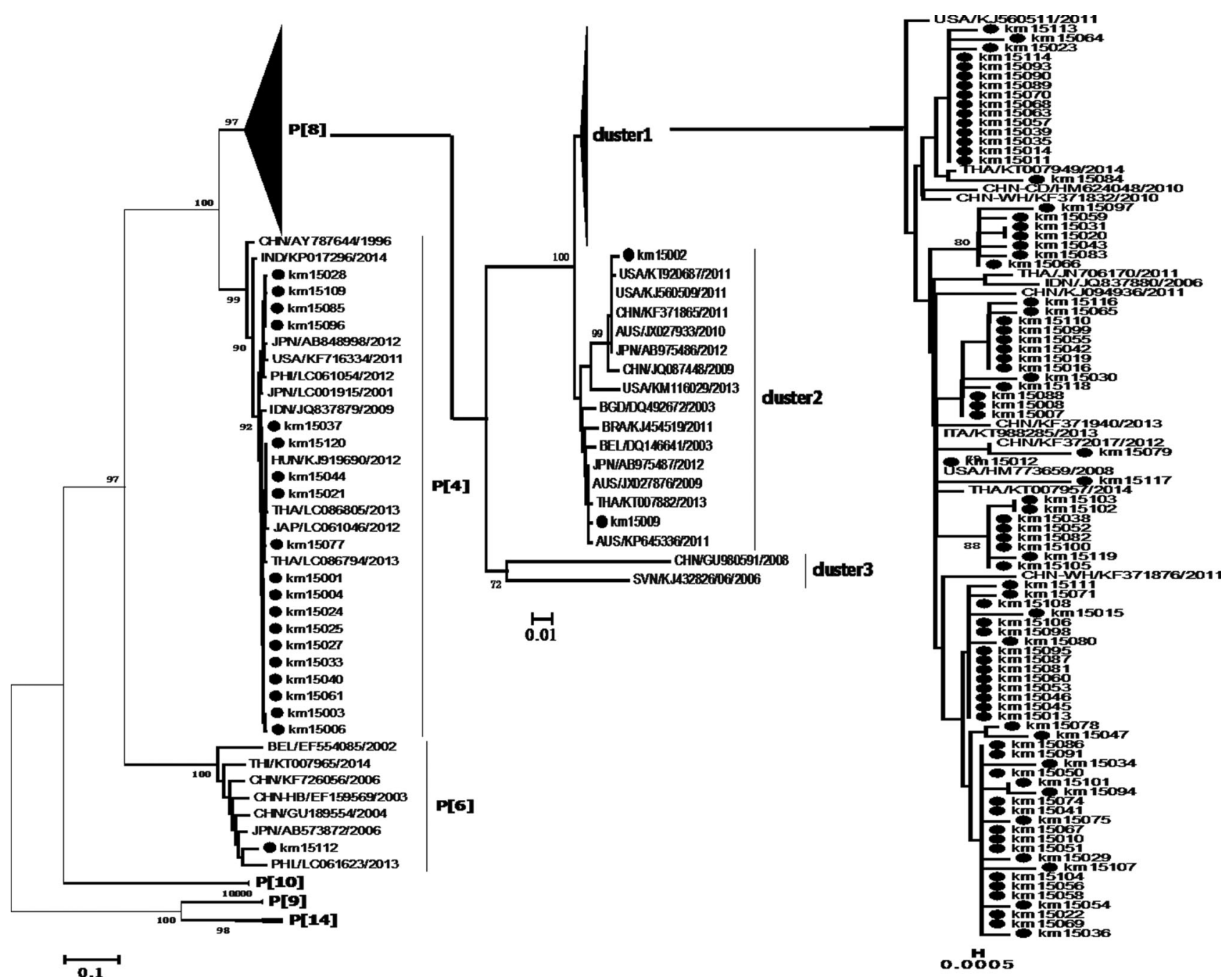

Fig. 2 Phylogenetic trees of VP4 sequences. A neighbor-joining (NJ) tree was conducted using MEGA 6.0 software with the Kimura 2-parameter model with gamma distribution. Bootstrap values were determined for 1000 repetitions. Kunming strains are indicated by black dots. The sequences of reference strains were obtained from GenBank
Table 1 The RVA genotypes in children of different age groups

\begin{tabular}{llllllll}
\hline Genotype & $0-12 \mathrm{M}$ & $13-24 \mathrm{M}$ & $25-36 \mathrm{M}$ & $37-48 \mathrm{M}$ & $49-60 \mathrm{M}$ & $61-168 \mathrm{M}$ & Total \\
\hline G9P[8] & 36 & 38 & 5 & 4 & 1 & 1 & 85 \\
G2P[4] & 1 & 6 & 4 & 3 & 1 & $\mathrm{~N}$ & 15 \\
G9P[4] & 1 & 2 & $\mathrm{~N}$ & $\mathrm{~N}$ & $\mathrm{~N}$ & 1 & 4 \\
G1P[8] & $\mathrm{N}$ & $\mathrm{N}$ & 1 & $\mathrm{~N}$ & $\mathrm{~N}$ & $\mathrm{~N}$ & 1 \\
G3P[8] & $\mathrm{N}$ & 1 & $\mathrm{~N}$ & $\mathrm{~N}$ & $\mathrm{~N}$ & $\mathrm{~N}$ & 1 \\
G4P[6] & $\mathrm{N}$ & 1 & $\mathrm{~N}$ & $\mathrm{~N}$ & $\mathrm{~N}$ & $\mathrm{~N}$ & 1 \\
\hline
\end{tabular}

The total number of rotavirus genotyped for both $\mathrm{G}$ and $\mathrm{P}$ was $107 . \mathrm{N}$, not detected amino acids of the G9P[8] strains to LLR, 14 amino acid (aa) substitutions were found in 28 aa (Table S4). In addition, alignments of the VP8* subunit sequences of G9P[8] strains to Rotarix ${ }^{\mathrm{TM}}$ and RotaTeq ${ }^{\mathrm{TM}}$ revealed that G9P[8] strains were more closely related to the latter, with four aa substitutions in eight aa. A low degree of homology was found between the VP8* subunit of G9P[8] and LLR, with 21 aa substitutions in 25 aa (Table S5). These findings suggest that these licensed vaccines may not be suitable for use in children in Kunming. LLR is licensed in China but is not included in national immunization programmes, and its effectiveness and safety are not known [23]. Effective vaccination programmes should be designed to reduce morbidity resulting from RVA infections. 
The high frequency of G9 strains observed in this study implies that this genotype is widespread at least in Kunming, and it may be potentially transmitted to other regions, as an important port for routes to Southeast Asian countries is located in Yunnan province. It is not clear whether the use of existing vaccines has resulted in a reduction in the distribution of other genotypes or if mutations have led to the immunological escape of this genotype. Further studies investigating the molecular basis of the transmission advantage of G9 strains are required.

Acknowledgments The authors would like to thank the clinical laboratories of the First People's Hospital of Yunnan Province and Kunming Children's Hospital.

\section{Compliance with ethical standards}

This study was reviewed and approved by the Institutional Ethical Committee of Kunming University of Science and Technology. The guardians of children participating in this study were informed of the aims of this investigation, and they provided oral consent.

Funding This study was supported by grants from the National Natural Science Foundation of China (81260248), National Science and Technology Sport Program of China (2014BAI01B01) and Innovation Talent Supporting Project (\#2015HC030) of Yunnan province.

Conflict of interest The authors declare that they have no conflict of interest.

Open Access This article is distributed under the terms of the Creative Commons Attribution 4.0 International License (http://creative commons.org/licenses/by/4.0/), which permits unrestricted use, distribution, and reproduction in any medium, provided you give appropriate credit to the original author(s) and the source, provide a link to the Creative Commons license, and indicate if changes were made.

\section{References}

1. Tate JE, Burton AH, Boschi-Pinto C, Parashar UD, World Health Organization-Coordinated Global Rotavirus Surveillance Network (2016) Global, regional, and national estimates of rotavirus mortality in children $<5$ years of age, 2000-2013. Clin Infect Dis 62(Suppl 2):S96-S105

2. Bishop RF, Davidson GP, Holmes IH, Ruck BJ (1973) Virus particles in epithelial cells of duodenal mucosa from children with acute non-bacterial gastroenteritis. Lancet 2(7841):1281-1283

3. Greenberg HB, Estes MK (2009) Rotaviruses: from pathogenesis to vaccination. Gastroenterology 136(6):1939-1951

4. Desselberger U (2014) Rotaviruses. Virus Res 190:75-96

5. Chen SC, Tan LB, Huang LM, Chen KT (2012) Rotavirus infection and the current status of rotavirus vaccines. J Formos Med Assoc 4:183-193

6. Matthijnssens J, Van Ranst M (2012) Genotype constellation and evolution of group A rotaviruses infecting humans. Curr Opin Virol 2(4):426-433

7. Duan ZJ, Liu N, Yang SH, Zhang J, Sun LW, Tang JY, Jin Y, Du ZQ, Xu J, Wu QB, Tong ZL, Gong ST, Qian Y, Ma JM, Liao XC, Widdowson MA, Jiang B, Fang ZY (2009) Hospital-based surveillance of rotavirus diarrhea in the People's Republic of China, August 2003-July 2007. J Infect Dis 200(Suppl 1):S167-S173
8. Orenstein EW, Fang ZY, Xu J, Liu C, Shen K, Qian Y, Jiang B, Kilgore PE, Glass RI (2007) The epidemiology and burden of rotavirus in China: a review of the literature from 1983 to 2005. Vaccine 25(3):406-413

9. Zhen Shuangping HY, Zongliu Hou (2006) Rotavirus molecular epidemic features in infants with enteritis in Kunming from 2002 to 2004. Chin J Pediatr 21(5):4

10. Aly M, Al Khairy A, Al Johani S, Balkhy H (2015) Unusual rotavirus genotypes among children with acute diarrhea in Saudi Arabia. BMC Infect Dis 15:192

11. Gouvea V, Glass RI, Woods P, Taniguchi K, Clark HF, Forrester B, Fang ZY (1990) Polymerase chain reaction amplification and typing of rotavirus nucleic acid from stool specimens. J Clin Microbiol 28(2):276-282

12. Abdel-Moneim AS, Al-Malky MI, Alsulaimani AA, Abuelsaad AS, Mohamed I, Ismail AK (2015) Sequence diversity of VP4 and VP7 genes of human rotavirus strains in Saudi Arabia. Foodborne Pathog Dis 12(12):937-944

13. Sai L, Sun J, Shao L, Chen S, Liu H, Ma L (2013) Epidemiology and clinical features of rotavirus and norovirus infection among children in Ji'nan, China. Virol J 10:302

14. Bonkoungou IJ, Damanka S, Sanou I, Tiendrebeogo F, Coulibaly SO, Bon F, Haukka K, Traore AS, Barro N, Armah GE (2011) Genotype diversity of group A rotavirus strains in children with acute diarrhea in urban Burkina Faso, 2008-010. J Med Virol 83(8): $1485-1490$

15. Mikami T, Nakagomi T, Tsutsui R, Ishikawa K, Onodera $\mathrm{Y}$, Arisawa K, Nakagomi O (2004) An outbreak of gastroenteritis during school trip caused by serotype $\mathrm{G} 2$ group A rotavirus. J Med Virol 73(3):460-464

16. Wang YH, Pang BB, Ghosh S, Zhou X, Shintani T, Urushibara N, Song YW, He MY, Liu MQ, Tang WF, Peng JS, Hu Q, Zhou DJ, Kobayashi N (2014) Molecular epidemiology and genetic evolution of the whole genome of G3P[8] human rotavirus in Wuhan, China, from 2000 through 2013. PLoS One 9(3):e88850

17. Unicomb LE, Podder G, Gentsch JR, Woods PA, Hasan KZ, Faruque AS, Albert MJ, Glass RI (1999) Evidence of high-frequency genomic reassortment of group A rotavirus strains in Bangladesh: emergence of type G9 in 1995. J Clin Microbiol 37(6): 1885-1891

18. Mullick S, Mandal P, Nayak MK, Ghosh S, De P, Rajendran K, Bhattacharya MK, Mitra U, Ramamurthy T, Kobayashi N, Chawla-Sarkar M (2014) Hospital based surveillance and genetic characterization of rotavirus strains in children $(<5$ years $)$ with acute gastroenteritis in Kolkata, India, revealed resurgence of G9 and G2 genotypes during 2011-2013. Vaccine 32(Suppl 1):A20A28

19. Clark HF, Hoshino Y, Bell LM, Groff J, Hess G, Bachman P, Offit PA (1987) Rotavirus isolate WI61 representing a presumptive new human serotype. J Clin Microbiol 25(9):1757-1762

20. Matthijnssens J, Heylen E, Zeller M, Rahman M, Lemey P, Van Ranst M (2010) Phylodynamic analyses of rotavirus genotypes G9 and G12 underscore their potential for swift global spread. Mol Biol Evol 27(10):2431-2436

21. Li Y, Wang SM, Zhen SS, Chen Y, Deng W, Kilgore PE, Wang $X Y$ (2014) Diversity of rotavirus strains causing diarrhea in $<5$ years old Chinese children: a systematic review. PLoS One 9(1):e84699

22. Wang X, Shi L, Qiao M, Wang Y, He M, Yong W, Du X, Guo B, Xie G, Xu Z, Ding J (2015) Phylogenetic Analyses and Characterization of the VP7 Genes of Serotype G9 Rotaviruses Circulating in Nanjing (China), 2012-2013. Bing du xue bao 31(4):425-432

23. Wang CM, Chen SC, Chen KT (2015) Current status of rotavirus vaccines. World J Pediatr 11(4):300-308 